\title{
SOME COMMENTS ON THE COMPOUND BINOMIAL MODEL
}

\author{
By David C.M. Dickson* \\ The University of Melbourne
}

\begin{abstract}
We show how ruin probabilities for the classical continuous time compound Poisson model can be approximated by ruin probabilities for a compound binomial model. We also discuss ruin related results for a compound binomial model with geometric claim amounts.
\end{abstract}

\section{KEYWORDS}

Ruin; compound binomial model; recursive calculation.

\section{INTRODUCTION}

GERBER (1988) presented some results for the compound binomial model which were analogues of results for the classical continuous time compound Poisson model. These results were further discussed by SHIU (1989). Willmot (1992) presented some explicit results for ultimate ruin probabilities for the compound binomial model.

In this note we derive some known results for the compound binomial model using very elementary methods. We also present results for a binomial claim numbers/geometric claim amounts model which correspond to results for the classical continuous time Poisson/exponential model. Our main purpose is to consider the conditions under which ultimate ruin probabilities for a compound binomial model give good approximations to ultimate ruin probabilities in the classical continuous time compound Poisson model.

We start by considering some basic results for a general discrete time risk model.

\section{A DISCRETE TIME RISK MODEL}

We will consider a risk model with the following characteristics:

(a) $X_{i}$ denotes the aggregate claim amount in the $i$-th time interval;

(b) $\left\{X_{i}\right\}_{i=1}^{x}$ is a sequence of independent and identically distributed random variables, each distributed on the non-negative integers;

(c) the insurer's premium income per unit time is 1 ;

(d) $E\left(X_{i}\right)<$ l.

* Part of this work was completed while the author was at Heriot-Watt University, Edinburgh. 
We will assume throughout that the insurer's initial surplus, denoted $u$, is an integer.

The insurer's surplus at time $t(t=1,2,3, \ldots)$ is denoted $Z(t)$ and given by

$$
Z(t)=u+t-\sum_{i=1}^{t} X_{i}
$$

The ultimate ruin probability for this model is defined by

$$
\psi(u)=\operatorname{Pr}[Z(t) \leq 0 \quad \text { for some } t, \quad t=1,2,3, \ldots]
$$

This definition corresponds to that given by GERBER (1988) but differs from that used by SHIU (1989) and WILlmot (1992). The reason for choosing this definition will become clear in Section 5. Note that ruin does not occur at time 0 if the initial surplus is zero. The survival probability is denoted $\delta(u)$ and $\delta(u)=1-\psi(u)$.

We define the severity of ruin function $G(u, y)$ for $u=0,1,2, \ldots$ and $y=1,2,3, \ldots$ by

$$
G(u, y)=\operatorname{Pr}[T<\infty \quad \text { and } \quad Z(T)>-y]
$$

where $T$ is the discrete time of ruin and is defined by

$$
\begin{aligned}
T & =\min \{t: Z(t) \leq 0, \quad t=1,2,3, \ldots\} \\
& =\infty \quad \text { if } \quad Z(t)>0 \quad \text { for } \quad t=1,2,3, \ldots
\end{aligned}
$$

Thus $G(u, y)$ represents the probability that ruin occurs and that the deficit at the time of ruin is at most $y-1$.

We denote by $b(k)$ and $B(k)$ respectively the probability function and distribution function of $X_{i}$.

\section{GENERAL RESULTS}

Result 1: For $u=1,2,3, \ldots$

$$
\delta(u)=\delta(0)+\sum_{k=1}^{u} \delta(k)[1-B(u-k)]
$$

Proof: By considering the possible aggregate claim amounts in the first time period we have that

$$
\delta(0)=b(0) \delta(1)
$$

and for $u=2,3,4, \ldots$

$$
\delta(u-1)=b(0) \delta(u)+\sum_{j=1}^{u-1} \delta(j) b(u-j)
$$


Hence, for $u=2,3,4, \ldots$

$$
\begin{aligned}
\sum_{k=0}^{u-1} \delta(k) & =b(0) \sum_{k=1}^{u} \delta(k)+\sum_{k=2}^{u} \sum_{j=1}^{k-1} \delta(j) b(k-j) \\
& =b(0) \sum_{k=1}^{u} \delta(k)+\sum_{k=1}^{u-1} \delta(k)[B(u-k)-b(0)] \\
& =b(0) \delta(u)+\sum_{k=1}^{u-1} \delta(k) B(u-k)
\end{aligned}
$$

Thus

$$
\begin{aligned}
b(0) \delta(u) & =\delta(0)+\sum_{k=1}^{u-1} \delta(k)[1-B(u-k)] \\
& =\delta(u-1)-\sum_{k=1}^{u-1} \delta(k) b(u-k)
\end{aligned}
$$

so that $\delta(u-1)=\delta(0)+\sum_{k=1}^{u-1} \delta(k)[1-B(u-1-k)]$

for $u=2,3,4, \ldots$, or equivalently,

$$
\delta(u)=\delta(0)+\sum_{k=1}^{u} \delta(k)[1-B(u-k)] \text { for } u=1,2,3, \ldots
$$

Result 2: The ruin probability from initial surplus zero is given by

$$
\psi(0)=E\left(X_{i}\right)
$$

Proof: For $y=0,1,2, \ldots$ define $g(0, y)$ to be the probability that ruin occurs from initial surplus zero and that the deficit at the time of ruin is $y$. Note that when the initial surplus is $u(>0), g(0, y)$ can be interpreted as the probability that the surplus falls below its initial level for the first time and by amount $y$. When $y=0$, $g(0, y)$ gives the probability that the surplus returns to its initial level for the first time without previously having been below its initial level. Using this interpretation we can write

$$
\delta(u)=\delta(0)+\sum_{y=1}^{u} g(0, u-y) \delta(y)
$$

The first term on the right hand side gives the probability that the surplus never falls below its initial level. For a fixed value of $y(<u), g(0, u-y) \delta(y)$ gives the probability that the surplus falls below its initial level for the first time to $y$ and that survival occurs from surplus level $y$. A similar interpretation applies when $y=u$. 
Summing over $y$ gives the probability that survival occurs and that the surplus process has not always been above its initial level.

By (3.1) we also have

$$
\delta(u)=\delta(0)+\sum_{y=1}^{u} \delta(y)[1-B(u-y)]
$$

Since equations (3.1) and (3.4) hold for $u=1,2,3, \ldots$, it follows that $g(0, y)=1-B(y)$. Equation (3.3) follows since

$$
\psi(0)=\sum_{y=0}^{\infty} g(0, y)
$$

If we write the premium income of 1 as $(1+\theta) E\left(X_{i}\right)$, then

$$
\psi(0)=1 /(1+\theta)
$$

as in the classical continuous time model.

We can easily obtain further ruin related results when the initial surplus is zero, starting with the joint distribution of the surplus prior to ruin and the deficit at ruin. We define a new function $f(u, x, y)$ for $x=1,2,3, \ldots$ and $y=0,1,2, \ldots$ as follows :

$$
f(u, x, y)=\operatorname{Pr}[T<\infty, Z(T)=-y \quad \text { and } \quad Z(T-1)=x]
$$

Thus $f(u, x, y)$ gives the probability that ruin occurs from initial surplus $u$, with a deficit of $y$ at the time of ruin and a surplus of $x$ one time unit prior to ruin. When $u=0$, the function is defined for $x=0,1,2, \ldots$, and $f(0,0, y)$ simply gives the probability that ruin occurs at time 1 with a deficit of $y$ at ruin. Thus $f(0,0, y)=b(y+1)$.

By considering the possible aggregate claim amounts in the first time period we can write

$$
f(u, x, y)=\sum_{j=0}^{u} b(j) f(u+1-j, x, y) \quad \text { for } \quad u=0,1,2, \ldots, x-1, x+1, \ldots
$$

and

$$
f(u, x, y)=\sum_{j=0}^{u} b(j) f(u+1-j, x, y)+b(x+y+1) \text { when } u=x
$$

Assuming that

$$
\sum_{u=0}^{\infty} f(u, x, y)<\infty
$$


we have that

$$
\begin{aligned}
\sum_{u=0}^{\infty} f(u, x, y) & =\sum_{u=0}^{\infty} \sum_{j=0}^{u} b(j) f(u+1-j, x, y)+b(x+y+1) \\
& =\sum_{u=1}^{\infty} f(u, x, y) \sum_{j=0}^{\infty} b(j)+b(x+y+1)
\end{aligned}
$$

Hence

$$
f(0, x, y)=b(x+y+1)
$$

As an immediate consequence of this we have that

$$
G(0, y)=\sum_{j=0}^{y-1} \sum_{x=0}^{\infty} b(x+j+1)=\sum_{j=0}^{y-1}[1-B(j)]
$$

and

$$
\psi(0)=\sum_{j=0}^{\infty}[1-B(j)]
$$

Similarly

$$
\begin{aligned}
\operatorname{Pr}[T<\infty \text { and } Z(t-1) \leq x-1 \mid u=0] & =\sum_{j=0}^{x-1} \sum_{y=0}^{\infty} b(j+y+1) \\
& =\sum_{j=0}^{x-1}[1-B(j)]=G(0, x)
\end{aligned}
$$

We have not discussed the conditions under which (3.6) holds. The most obvious situation when (3.6) holds is when Lundberg's inequality applies. Formula (3.7) does however hold when the sum in (3.6) is infinite.

The results presented above are in terms of a general distribution $B(k)$. However, they are in fact the same as results given by GerBER (1988) and SHIU (1989). This follows since the distribution of $X_{i}$ can be expressed as a compound binomial distribution with binomial parameters 1 and $1-b(0)$ and probability function for individual claims $b(j) /[1-b(0)]$ for $j=1,2,3, \ldots$.

\section{THE BINOMIAL/GEOMETRIC MODEL}

Throughout this section we assume that the distribution of the number of claims per unit time is binomial with parameters 1 and $p$, and the individual claim amount distribution is geometric with distribution function $P(x)$ and probability function

$$
p(x)=(1-\alpha) \alpha^{x-1} \quad \text { for } \quad x=1,2,3, \ldots
$$

Then

$$
B(k)=1-p \alpha^{k} \quad \text { for } \quad k=0,1,2, \ldots
$$


Since we have assumed that $E\left(X_{i}\right)<1$, the parameters $p$ and $\alpha$ must be such that $p /(1-\alpha)<1$.

We can rewrite equation (3.1) as

$$
\psi(u)=\psi(0)-\sum_{k=1}^{u}[1-\psi(k)][1-B(u-k)]
$$

and inserting for $\psi(0)$ we have

$$
\psi(u)=\sum_{k=1}^{u} \psi(k)[1-B(u-k)]+\sum_{k=u}^{\infty}[1-B(k)]
$$

The continuous time compound Poisson analogue of this equation can be found in, for example, GERBER (1979).

Now insert for $B(k)$ to find that

$$
\psi(u)=\sum_{k=1}^{u} \psi(k) p \alpha^{u-k}+\sum_{k=u}^{\infty} p \alpha^{k}
$$

and

$$
\psi(u+1)=\sum_{k=1}^{u+1} \psi(k) p \alpha^{u+1-k}+\sum_{k=u+1}^{\infty} p \alpha^{k}
$$

If we multiply (4.1) by $\alpha$, subtract from (4.2) and rearrange we find that

$$
\psi(u+1)-\frac{\alpha}{1-p} \psi(u)=0
$$

The solution to this difference equation is

$$
\psi(u)=c\left(\frac{\alpha}{1-p}\right)^{u}
$$

from which it follows that $c=\psi(0)$. In fact, we can write $\psi(u)=\psi(0) \exp \{-R u\}$, where $R$ is the adjustment coefficient for this process. $R$ is the unique positive number satisfying

$$
E\left[\exp \left\{R\left(X_{i}-1\right)\right\}\right]=1
$$

and it is an elementary exercise to show that for this model $\exp \{R\}=(1-p) / \alpha$. Thus we have a complete analogy with the form of the ruin probability for the Poisson/exponential model which can be written in exactly the same way. (See, for example, GERBER (1979)). We note that this solution matches that given by WiLlmot (1992) for $\delta(u)$, allowing for different definitions of ruin/survival.

We now extend the analogy to the severity of ruin. We can use the function $g(0, y)$ to write down an equation for $G(u, y)$ by considering the first occasion on which the surplus falls below (or returns to without previously having been below) its initial level. 
We have

$$
\begin{aligned}
G(u, y) & =\sum_{k=u}^{u+y-1} g(0, k)+\sum_{k=0}^{u-1} g(0, k) G(u-k, y) \\
& =\sum_{k=u}^{u+y-1} g(0, k)+\sum_{k=1}^{u} g(0, u-k) G(k, y)
\end{aligned}
$$

Now insert $g(0, k)=1-B(k)=p \alpha^{k}$ to give

$$
G(u, y)=\sum_{k=u}^{u+y-1} p \alpha^{k}+\sum_{k=1}^{u} p \alpha^{u-k} G(k, y)
$$

and

$$
G(u+1, y)=\sum_{k=u+1}^{u+y} p \alpha^{k}+\sum_{k=1}^{u+1} p \alpha^{u+1-k} G(k, y)
$$

Using the same technique as before we find that

$$
G(u+1, y)-\frac{\alpha}{1-p} G(u, y)=0
$$

and hence

$$
G(u, y)=G(0, y)\left(\frac{\alpha}{1-p}\right)^{u}
$$

Finally

$$
G(0, y)=\sum_{k=0}^{y-1} g(0, k)=\sum_{k=0}^{y-1} p \alpha^{k}=p \frac{1-\alpha^{y}}{1-\alpha} \quad \text { for } \quad y=1,2,3, \ldots
$$

and so we can write

$$
G(u, y)=\left(1-\alpha^{y}\right) \frac{p}{1-\alpha}\left(\frac{\alpha}{1-p}\right)^{u}=P(y) \psi(u)
$$

Thus the form of $G(u, y)$ is identical to that for the Poisson/exponential model. (See, for example, DicKSON (1992)). However, unlike the Poisson/exponential model, the distribution of the deficit at the time of ruin is not identical to the individual claim amount distribution. The deficit is geometrically distributed with parameter $\alpha$, but on $0,1,2, \ldots$, since $G(u, y) / \psi(u)$ gives the probability that the deficit is less than or equal to $y-1$, given that ruin occurs, and so

$$
\operatorname{Pr}[-Z(T)<y \mid T<\infty]=1-\alpha^{y} \quad \text { for } \quad y=1,2,3, \ldots
$$

Let us now consider the situation when $u=0$ further. We have already noted that the deficit at the time of ruin is geometrically distributed on $0,1,2, \ldots$ with parameter $\alpha$, and by (3.8) the distribution of the surplus at time $T-1$ is the same. 
The conditional probability function of the deficit at $T$ and of the surplus at $T-1$, conditioning on the event that ruin occurs, is

$$
\tilde{g}(0, x)=(1-\alpha) \alpha^{x}, \quad x=0,1,2, \ldots
$$

If we consider the conditional distribution of the surplus one time unit before ruin and of the deficit at ruin, conditioning on the event that ruin occurs, and again use a tilde to denote a conditional probability, then

$$
\tilde{f}(0, x, y)=\frac{b(x+y+1)}{\psi(0)}=\frac{p(1-\alpha) \alpha^{x+y}}{p /(1-\alpha)}=\tilde{g}(0, x) \tilde{g}(0, y)
$$

so that, conditionally, the surplus one time unit before ruin and the deficit at ruin are independent and identically distributed. This situation also exists in the Poisson/exponential model where the surplus prior to ruin and deficit at ruin are independent, identically distributed variables, and the conditional distribution of the claim causing ruin is Gamma(2).

Finally, if we define the conditional probability function of the claim causing ruin as $h(0, z)$ for $z=1,2,3, \ldots$ then

$$
h(0, z)=\sum_{x=0}^{z-1} \tilde{f}(0, x, z-x-1)=\sum_{x=0}^{z-1}(1-\alpha)^{2} \alpha^{z-1}=z(1-\alpha)^{2} \alpha^{z-1}
$$

The conditional distribution of the claim causing ruin is thus negative binomial with parameters 2 and $1-\alpha$, shifted one unit to the right.

\section{CALCULATION OF RUIN PROBABILITIES}

GERBER (1988) states that the compound binomial model can be used to approximate the continuous time compound Poisson model. In this section we investigate this statement by considering ultimate ruin probabilities.

To calculate ruin probabilities for the compound binomial model, we will adapt the framework described by Dickson and WATERs (1991, Sections 1 and 8) who use a discrete time compound Poisson model to approximate a classical continuous time compound Poisson model under which both the Poisson parameter and mean individual claim amount are 1. The characteristics of this model are as follows:

(a) individual claim amounts are distributed on the non-negative integers with mean $\beta$, where $\beta(>1)$ is an integer;

(b) the Poisson parameter for the expected number of claims per unit time is $1 /[(1+\theta) \beta]$

(c) the premium income per unit time is 1 .

We will replace this discrete compound Poisson model by a compound binomial model. We simply change (b), replacing the Poisson distribution by a binomial distribution with parameters 1 and $1 /[(1+\theta) \beta]$. For reasons given by DICKSON and WATERS (1991) we can regard $\psi(\beta u)$ as an approximation to $\psi_{c}(u)$, the ultimate ruin probability for the continuous compound Poisson model. Note that the definition of $\psi(u)$ given in Section 2 corresponds to that used in this approxima- 
tion. In effect all we are doing is approximating a discrete compound Poisson model (which approximates a continuous compound Poisson model) by a compound binomial model. The approximation to the discrete compound Poisson model is reasonable for large values of $\beta$, since the Poisson distribution with parameter $1 /[(1+\theta) \beta]$ is then very close to the approximating binomial distribution. For example, if $\beta=100$ and $\theta=0.1$, then the probability of more than one claim per unit time under the compound Poisson model is 0.00004 . Note that there is one small difference between this formulation of the compound binomial model and that used by previous authors. In this formulation, individual claim amounts are distributed on the non-negative integers rather than the positive integers. The reason for this is simply that in order to approximate ruin probabilities in the classical continuous time compound Poisson model, we have to discretize the continuous individual claim amount distribution in that model. In our first two examples, we will use the discretization proposed by DE VYLDER and GOOVAERTS (1988), which discretizes the distribution on the non-negative integers. If we had chosen a discretization on the positive integers then our model would correspond to that used by previous authors.

We will calculate ruin probabilities recursively from the formulae

$$
\psi(1)=b(0)^{-1}[\psi(0)-1+B(0)]
$$

and for $u=2,3,4, \ldots$

$$
\psi(u)=b(0)^{-1}\left[\psi(u-1)-1+B(u-1)-\sum_{j=1}^{u-1} b(j) \psi(u-j)\right]
$$

These formulae correspond to Gerber's (1988) formulae (5) and (6). In each of the following examples the premium loading factor, $\theta$, is $10 \%$.

Example 1: Let the individual claim amount distribution in the continuous time model be exponential with mean 1. Then it is well known (see, for example, GERBER (1979)) that

$$
\psi_{r}(u)=\frac{1}{1+\theta} \exp \left(-R_{c} u\right) \quad \text { where } \quad R_{c}=\theta /(1+\theta)
$$

Table 1 shows exact and approximate values of $\psi_{c}(u)$. The approximate values are calculated from (3.5), (5.1) and (5.2). The legend for this table is as follows :

(1) denotes the exact value of $\psi_{c}(u)$;

(2) denotes the approximate value when $\beta=50$;

(3) denotes the ratio of the value in (2) to that in (1);

(4) denotes the approximate value when $\beta=100$;

(5) denotes the ratio of the value in (4) to that in (1);

(6) denotes the approximate value when $\beta=200$;

(7) denotes the ratio of the value in (6) to that in (1). 
TABLE 1

(See Example 1 for details)

\begin{tabular}{l|ccccccc}
\hline \hline & $(1)$ & $(2)$ & $(3)$ & $(4)$ & $(5)$ & $(6)$ & $(7)$ \\
\hline$u=0$ & 0.9091 & 0.9091 & 1.0000 & 0.9091 & 1.0000 & 0.9091 & 1.0000 \\
$u=2$ & 0.7580 & 0.7567 & 0.9983 & 0.7573 & 0.9992 & 0.7576 & 0.9996 \\
$u=4$ & 0.6319 & 0.6299 & 0.9967 & 0.6309 & 0.9983 & 0.6314 & 0.9992 \\
$u=6$ & 0.5269 & 0.5243 & 0.9950 & 0.5256 & 0.9975 & 0.5262 & 0.9988 \\
$u=8$ & 0.4393 & 0.4364 & 0.9934 & 0.4378 & 0.9967 & 0.4386 & 0.9983 \\
$u=10$ & 0.3663 & 0.3632 & 0.9917 & 0.3647 & 0.9959 & 0.3655 & 0.9979 \\
$u=20$ & 0.1476 & 0.1451 & 0.9835 & 0.1463 & 0.9917 & 0.1470 & 0.9959 \\
$u=40$ & 0.0240 & 0.0232 & 0.9673 & 0.0236 & 0.9836 & 0.0238 & 0.9918 \\
$u=80$ & 0.0006 & 0.0006 & 0.9357 & 0.0006 & 0.9674 & 0.0006 & 0.9836 \\
\hline
\end{tabular}

We note the following points about Table 1 :

(a) When $u>0$, the approximate values are less than the exact ones. This is to be expected since the compound binomial model excludes the possibility of multiple claims per unit time.

(b) As the value of $\beta$ increases, the approximate values become closer to the exact ones. This is as expected for reasons given by Dickson and WATERS (1991, Section 2).

(c) The larger the value of $u$, the poorer the approximation becomes.

(d) Even with a large value of $\beta$, the approximate values do not always agree with the exact values to four decimal places.

Example 2: Let the individual claim amount distribution in the continuous time model be Pareto with parameters 2 and 1. Table 2 shows exact and approximate values of $\psi_{c}(u)$. (The exact values have been calculated using DickSON and WATERS' (1991) algorithm and are "exact" at least to three decimal places). The legend for Table 2 is the same as for Table 1. The only additional comment that we would make about Table 2 is that, for the same magnitude of ruin probability, the approximate values are slightly closer to the exact values than in Example 1.

TABLE 2

(SeE Example 2 for details)

\begin{tabular}{l|ccccccc}
\hline \hline & $(1)$ & $(2)$ & $(3)$ & $(4)$ & $(5)$ & $(6)$ & $(7)$ \\
\hline$u=0$ & 0.9091 & 0.9091 & 1.0000 & 0.9091 & 1.0000 & 0.9091 & 1.0000 \\
$u=2$ & 0.8102 & 0.8097 & 0.9994 & 0.8100 & 0.9997 & 0.8101 & 0.9998 \\
$u=4$ & 0.7498 & 0.7491 & 0.9991 & 0.7494 & 0.9996 & 0.7496 & 0.9998 \\
$u=6$ & 0.7021 & 0.7014 & 0.9990 & 0.7018 & 0.9995 & 0.7020 & 0.9997 \\
$u=8$ & 0.6620 & 0.6613 & 0.9989 & 0.6617 & 0.9994 & 0.6619 & 0.9997 \\
$u=10$ & 0.6271 & 0.6264 & 0.9988 & 0.6267 & 0.9994 & 0.6269 & 0.9997 \\
$u=20$ & 0.4981 & 0.4974 & 0.9985 & 0.4978 & 0.9992 & 0.4980 & 0.9996 \\
$u=40$ & 0.3479 & 0.3473 & 0.9982 & 0.3476 & 0.9991 & 0.3477 & 0.9995 \\
$u=80$ & 0.2040 & 0.2036 & 0.9981 & 0.2038 & 0.9990 & 0.2039 & 0.9995 \\
\hline
\end{tabular}


In Section 4 we discussed the binomial/geometric model as the discrete analogue of the Poisson/exponential model. In Example 3 we illustrate how ruin probabilities for the binomial/geometric model can be used to approximate those for the Poisson/exponential model. We have included this example purely for interest as the approach does not generalise to other compound Poisson models.

Example 3: We will use the same framework as in Examples 1 and 2, but will discretize the exponential individual claim amount distribution as a geometric distribution with mean $\beta$. This discretization is a reasonable one for large values of $\beta$ since when $\beta$ is large

$$
P(x)=1-\left(1-\beta^{-1}\right)^{x} \approx 1-\exp \{-x / \beta\} \quad \text { for } \quad x=0,1,2, \ldots
$$

As noted in Section 4 , for the geometric individual claim amount distribution,

$$
\psi(\beta u)=\frac{1}{1+\theta} \exp (-R \beta u) \quad \text { where } \quad R=\log _{e}\left(\frac{(1+\theta) \beta-1}{(1+\theta)(\beta-1)}\right)
$$

It is easy to show that

$$
\lim _{\beta \rightarrow \infty} \beta R=\frac{\theta}{1+\theta}
$$

so that for large values of $\beta, \psi(\beta u)$ should give a good approximation to $\psi_{c}(u)$.

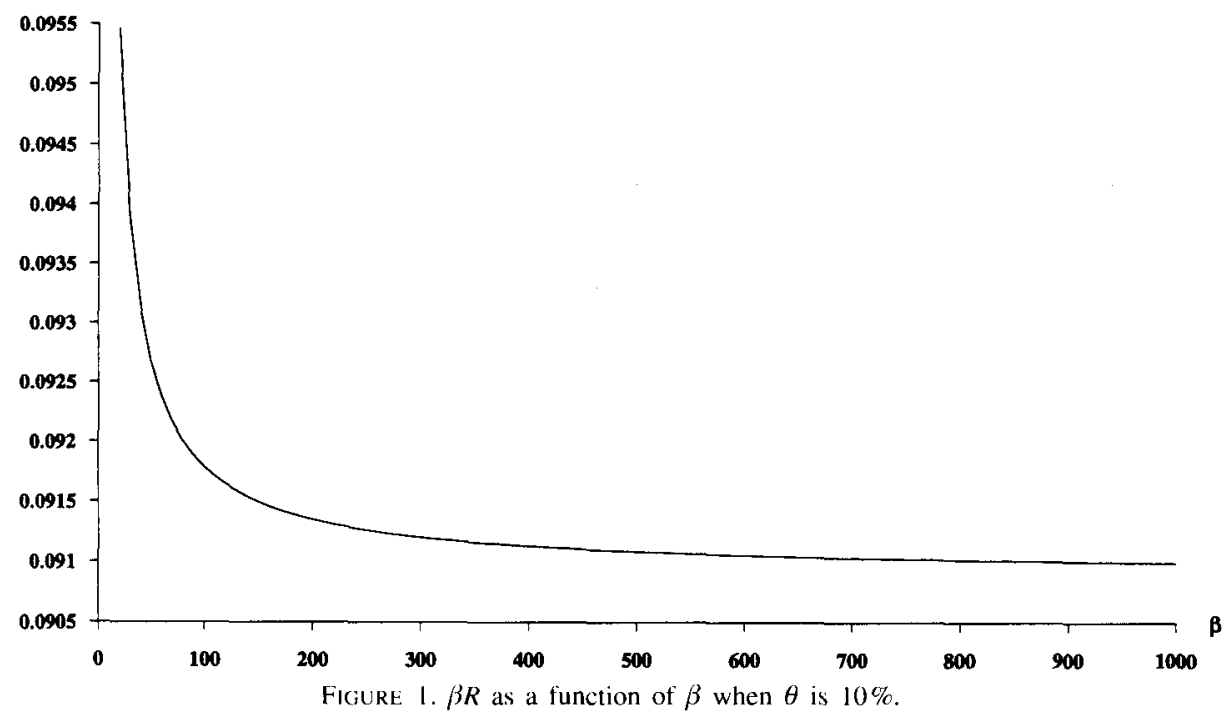

Figure 1 shows the function $\beta R$ (when $\theta$ is $10 \%$ ) and Table 3 shows exact and approximate values of $\psi_{c}(u)$. The legend for Table 3 is as follows:

(1) denotes the exact value of $\psi_{c}(u)$;

(2) denotes the approximate value when $\beta=100$; 
(3) denotes the ratio of the value in (2) to that in (1);

(4) denotes the approximate value when $\beta=1,000$;

(5) denotes the ratio of the value in (4) to that in (1);

(6) denotes the approximate value when $\beta=10,000$;

(7) denotes the ratio of the value in (6) to that in (1).

TABLE 3

(SEE EXAMPLE 3 FOR DeTAIls)

\begin{tabular}{l|ccccccc}
\hline \hline & $(1)$ & $(2)$ & $(3)$ & $(4)$ & $(5)$ & $(6)$ & $(7)$ \\
\hline$u=0$ & 0.9091 & 0.9091 & 1.0000 & 0.9091 & 1.0000 & 0.9091 & 1.0000 \\
$u=2$ & 0.7580 & 0.7566 & 0.9982 & 0.7578 & 0.9998 & 0.7579 & 1.0000 \\
$u=4$ & 0.6319 & 0.6297 & 0.9965 & 0.6317 & 0.9997 & 0.6319 & 1.0000 \\
$u=6$ & 0.5269 & 0.5241 & 0.9948 & 0.5266 & 0.9995 & 0.5269 & 0.9999 \\
$u=8$ & 0.4393 & 0.4362 & 0.9930 & 0.4390 & 0.9993 & 0.4393 & 0.9999 \\
$u=10$ & 0.3663 & 0.3631 & 0.9913 & 0.3659 & 0.9991 & 0.3662 & 0.9999 \\
$u=20$ & 0.1476 & 0.1450 & 0.9826 & 0.1473 & 0.9983 & 0.1475 & 0.9998 \\
$u=40$ & 0.0240 & 0.0231 & 0.9656 & 0.0239 & 0.9965 & 0.0239 & 0.9997 \\
$u=80$ & 0.0006 & 0.0006 & 0.9323 & 0.0006 & 0.9931 & 0.0006 & 0.9993 \\
\hline
\end{tabular}

Table 3 shows the same features as Tables 1 and 2. The great advantage of using the geometric discretization is that approximate values for $\psi_{c}(u)$ can be calculated from a formula. This allows us to use very large values for $\beta$, and shows that even with a large value of $\beta$ (i.e. 10,000) the approximate values do not all match the exact ones to four decimal places. By contrast, if $b(x)$ and $B(x)$ in (5.1) and (5.2) are values from a compound Poisson distribution, then a relatively small value of $\beta$ produces the same degree of accuracy. (See, for example, DickSON and WATERS (1991, Table 5)).

We conclude that it is possible to successfully approximate ruin probabilities for the classical continuous time compound Poisson model by those for a compound binomial model. The main advantage in using the compound binomial model is that it is not necessary to perform recursive calculations to find the probability function $b(x)$ to use formulae (5.1) and (5.2). However, this advantage is outweighed by the fact that a large value of $\beta$ is required when using the compound binomial model in order to obtain a good approximation to $\psi_{c}(u)$.

\section{ACKNOWLEDGEMENT}

I am grateful to the referees and editor for comments on the first draft of this paper.

\section{REFERENCES}

DE Vylder, F. and Goovaerts, M.J. (1988) Recursive calculation of finite-time ruin probabilities. Insurance: Mathematics and Economics 7, 1-8.

Dickson, D.C.M. and WATERS, H.R. (1991) Recursive calculation of survival probabilities. ASTIN Bulletin 21, 199-221. 
Dickson, D.C.M. (1992) On the distribution of the surplus prior to ruin. Insurance: Mathematics and Economics 11, 191-207.

Gerber, H. U. (1979) An Introduction to Mathematical Risk Theory. S.S. Huebner Foundation Monograph Series No. 8. Distributed by R. Irwin, Homewood, IL.

Gerber, H. U. (J988) Mathematical fun with the compound binomial process. ASTIN Bulletin 18, $161-168$

SHIU, E.S.W. (1989) The probability of eventual ruin in the compound binomial model. ASTIN Bulletin 19. $179-190$.

WIL.LMot, G.E. (1992) Ruin probabilities in the compound binomial model. Insurance: Mathematics and Economics 12, 133-142.

David C. M. Dickson

Centre for Actuarial Studies, Faculty of Economics and Commerce, The University of Melbourne, Parkville, Victoria 3052, Australia.

February 1993

Revised November 1993 